\title{
A non-uniform distribution of the nearest brown dwarfs
}

\author{
G. Bihain and R.-D. Scholz
}

\author{
Leibniz-Institut für Astrophysik Potsdam (AIP), An der Sternwarte 16, 14482 Potsdam, Germany \\ e-mail: [gbihain;rdscholz]@aip.de
}

Received 18 December 2015 / Accepted 23 February 2016

\section{ABSTRACT}

\begin{abstract}
Context. The census of solar neighbours is still complemented by new discoveries, mainly of very low-mass, faint dwarfs, close to or within the substellar domain. These discoveries contribute to a better understanding of the field population; its origin in terms of Galactic dynamics and (sub)stellar formation and evolution. Also, the nearest stars and brown dwarfs at any given age allow the most precise direct characterization, including the search for planetary companions.

Aims. We aim to further assess the substellar census on the Galactic plane.

Methods. We projected the 136 stars and 26 brown dwarfs known at $<6.5 \mathrm{pc}$ on the Galactic plane and evaluated their distributions. Results. Stars present a uniform- and brown dwarfs a non-uniform distribution, with 21 objects behind the Sun and only five ahead relative to the direction of rotation of the Galaxy. This substellar configuration has a probability of $0.098_{-0.098}^{+10.878} \%$ relative to uniformity. The helio- and geocentric nature of the distribution suggests it might result in part from an observational bias, which if compensated for by future discoveries, might increase the brown-dwarf-to-star ratio, shifting it closer to values found in some star forming regions.
\end{abstract}

Key words. astrometry - proper motions - stars: distances - stars: kinematics and dynamics - brown dwarfs - solar neighborhood

\section{Introduction}

The stellar neighbourhood of the Sun comprises main sequence stars in the majority and a minority of cool white dwarfs (see RECONS $^{1}$ census) spanning ages of $\sim 1-10$ Gyr (Giammichele et al. 2012). At $<6.5 \mathrm{pc}$, the stellar census is expected to be relatively complete with a few new white- or low-mass dwarfs to be discovered. The first substellar objects identified at $<6.5 \mathrm{pc}$ were GJ 229 B, a methane dwarf (T7V; Nakajima et al. 1995; Oppenheimer et al. 1995), and LP 944-020, a lithium brown dwarf (M9; Tinney 1998). Searches based on the Two Micron All Sky Survey (2MASS, Skrutskie et al. 2006) and Wide-field Infrared Survey Explorer (WISE, Wright et al. 2010) as well as other surveys have incremented the number of brown dwarfs (see census by Kirkpatrick et al. 2012). Star forming regions appear to produce free-floating substellar objects in abundance, even at planetary masses (Béjar et al. 2001; Peña Ramírez et al. 2012, 2016; Mužić et al. 2015); furthermore, the lower their masses, the higher their velocity dispersions can be (Zapatero Osorio et al. 2014). This explains why numerous faint and (infra)red relics of such objects are indeed found in the solar vicinity in the form of ultra-cool, late-T- and early-Y-type dwarfs, with the 3-10 $M_{\text {jup }}$ WISE 0855-0714 as the most spectacular example (Luhman 2014b). To further assess the substellar census, we consider the distribution of known objects on the Galactic plane.

\section{Data analysis and results}

We updated the RECONS list (as of 2012 January 1) of the 100 nearest systems $(<6.5 \mathrm{pc})$ and the $8 \mathrm{pc}$ sample by Kirkpatrick et al. (2012) with new objects or improved parallaxes or spectral types. At $<6.5 \mathrm{pc}, 16$ brown dwarfs (Table 1 ) are not in RECONS yet. (The RECONS census is restricted to objects with trigonometric parallax errors smaller than 10 mas.) Similarly, eight stars at $<6.5 \mathrm{pc}$ are not in RECONS. WISE J1540-5101 (M7;

REsearch Consortium On Nearby Stars, www . recons .org
Table 1. Brown dwarfs known at $<6.5 \mathrm{pc}$.

\begin{tabular}{|c|c|c|c|c|c|}
\hline Object & $\begin{array}{c}l \\
\text { (deg) }\end{array}$ & $\begin{array}{c}b \\
\text { (deg) }\end{array}$ & $\begin{array}{l}\text { Plx } \\
\text { (mas) }\end{array}$ & SpT & Ref. \\
\hline WISE J1049-5319 A & 285.18 & 5.29 & $496 \pm 37$ & L7.5 & 1,2 \\
\hline WISE J1049-5319 B & 285.18 & 5.29 & $496 \pm 37$ & T0.5 & 1,2 \\
\hline WISE J0855-0714 & 234.99 & 23.35 & $433 \pm 15$ & $>\mathrm{Y} 2$ & 3,4 \\
\hline eps Indi $\mathrm{Ba}^{*}$ & 336.12 & -48.15 & $276.06 \pm 0.28$ & $\mathrm{~T} 1$ & 5,6 \\
\hline eps Indi $\mathrm{Bb}^{*}$ & 336.12 & -48.15 & $276.06 \pm 0.28$ & T6 & 5,6 \\
\hline SCR J1845-6357 B* & 331.52 & -23.50 & $259.50 \pm 1.11$ & T6 & 7,8 \\
\hline UGPS J0722-0540 & 221.54 & 4.27 & $242.80 \pm 24$ & T9 & 9,10 \\
\hline WISE J0350-5658 & 269.01 & -46.67 & $238 \pm 38$ & Y1 & 11,11 \\
\hline DENIS J0817-6155 & 276.06 & -14.40 & $203 \pm 13$ & T6 & 12,12 \\
\hline WISE J1639-6847 & 321.20 & -14.48 & $202.3 \pm 3.5$ & Y0 & 13,14 \\
\hline DENIS J0255-4700* & 260.59 & -58.67 & $201.37 \pm 3.89$ & L9 & 15,16 \\
\hline WISE J0521+1025 & 192.82 & -14.62 & $200 \pm 52$ & $\mathrm{~T} 7.5$ & 17,17 \\
\hline WISE J1506+7027 & 108.27 & 42.61 & $193 \pm 26$ & T6 & 11,18 \\
\hline 2MASS J0939-2448* & 256.91 & 20.47 & $187.30 \pm 4.60$ & $\mathrm{~T} 8$ & 19,19 \\
\hline WISE J1741+2553 & 50.11 & 26.10 & $180 \pm 15$ & T9 & 20,21 \\
\hline 2MASS J1114-2618 & 277.39 & 31.73 & $179.20 \pm 1.4$ & $\mathrm{~T} 7.5$ & 22,16 \\
\hline 2MASS J0415-0935* & 202.94 & -38.94 & $175.20 \pm 1.70$ & $\mathrm{~T} 8$ & 22,16 \\
\hline WISE J1541-2250 & 346.55 & 25.26 & $175.1 \pm 4.4$ & Y0.5 & 13,11 \\
\hline GJ 229 B* $^{*}$ & 228.61 & -18.44 & $173.81 \pm 0.99$ & $\mathrm{~T} 7$ & 5,16 \\
\hline GJ $570 \mathrm{D}^{*}$ & 338.23 & 32.75 & $171.22 \pm 0.94$ & $\mathrm{~T} 7.5$ & 5,16 \\
\hline WISE J0720-0846 B & 224.01 & 2.29 & $166 \pm 28$ & T5.5 & 23,24 \\
\hline 2MASS J0937+2931* & 197.85 & 47.58 & $163.39 \pm 1.76$ & T6 & 25,16 \\
\hline WISE J0410+1502 & 177.95 & -25.92 & $160 \pm 9$ & Y0 & 26,27 \\
\hline 2MASS J1503+2525 & 37.19 & 60.47 & $157.2 \pm 2.2$ & T5 & 22,16 \\
\hline SIMP J0136+0933 & 141.22 & -51.69 & $156 \pm 7$ & $\mathrm{~T} 2.5$ & 28,28 \\
\hline LP 944-020* & 236.67 & -53.41 & $155.89 \pm 1.03$ & M9 & 29,30 \\
\hline
\end{tabular}

Notes. ${ }^{(*)}$ In RECONS.

References. References for parallaxes and spectral types are: (1) Luhman (2013); (2) Burgasser et al. (2013); (3) Luhman \& Esplin (2014); (4) Leggett et al. (2015); (5) van Leeuwen (2007); (6) McCaughrean et al. (2004); (7) Henry et al. (2006); (8) Kasper et al. (2007); (9) Leggett et al. (2012); (10) Lucas et al. (2010); (11) Kirkpatrick et al. (2012); (12) Artigau et al. (2010); (13) Tinney et al. (2014); (14) Schneider et al. (2015); (15) Costa et al. (2006); (16) Burgasser et al. (2006); (17) Bihain et al. (2013); (18) Kirkpatrick et al. (2011); (19) Burgasser et al. (2008); (20) Dupuy \& Kraus (2013); (21) Scholz et al. (2011); (22) Dupuy \& Liu (2012); (23) Burgasser et al. (2015a); (24) Burgasser et al. (2015b); (25) Schilbach et al. (2009); (26) Beichman et al. (2014); (27) Cushing et al. (2011); (28) Artigau et al. (2006); (29) Dieterich et al. (2014); (30) Henry et al. (2004). 

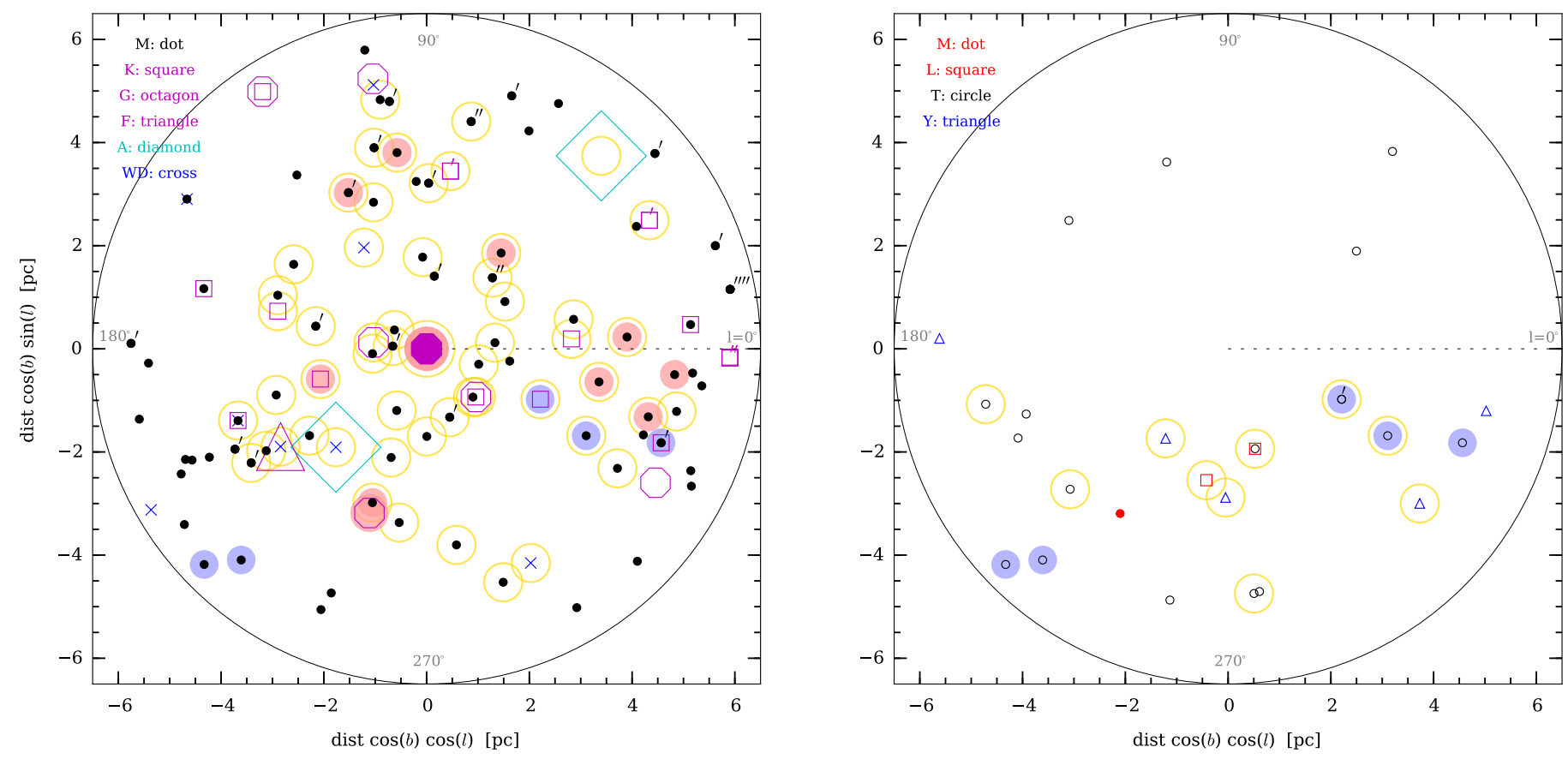

Fig. 1. Distribution of $<6.5 \mathrm{pc}$ stars (left panel) and brown dwarfs (right panel) on the Galactic coordinates plane. M, K, G, F, A, and WD type stars are represented by dots, squares, octagons, triangles, diamonds, and crosses, and M, L, T, and Y type brown dwarfs are represented by dots, squares, circles, and triangles, respectively. The filled octagon indicates the Sun and the dotted line the direction towards the Galactic centre. Different object symbols centred on the same location are multiple systems; a prime indicates one more component of the same spectral type. Symbols with blue and pink surroundings are star-brown dwarf and star-planet systems, respectively. Single objects or multiple systems nearer than 5.16 pc (half-volume distance) are indicated with a yellow circle.

Kirkpatrick et al. 2014; Pérez Garrido et al. 2014), TYC 39801081-1 ( M3.5; Finch et al. 2014), and WISE J0720-0846 AB (M9.5+T5; Scholz 2014; Ivanov et al. 2015; Burgasser et al. 2015a,b) are three recently discovered stars. Both 2MASS J0533-4257 (M4.5) and 2MASS J1845-1409 AB (M5+M5) have provisional distances (Kirkpatrick et al. 2012). Finally, G 161-71 and L 43-72 (M6 and M5 as from Gaidos et al. 2014), already mentioned as nearby stars by McCaughrean et al. (2002) and Scholz et al. (2005), respectively, have updated distances (Winters et al. 2015). Compared to the sample of Kirkpatrick et al. (2012), at $<6.5$ pc there are six new brown dwarfs (WISE J1049-5319 AB, WISE J0855-0714, WISE J1639-6847, WISE J0521+1025, WISE J0720-0846 $\mathrm{B})$, and owing to more accurate parallaxes, one brown dwarf shifting in (2MASS J1114-2618), three shifting out but at $<8$ pc (WISE J2056+1459, WISE J1405+5534, and WISE J0254+0223; see references below), and two shifting out at $>8$ pc (WISE J0146+4234 and WISE J0535-7500; trigonometric parallaxes from Beichman et al. 2014 and Tinney et al. 2014, respectively).

We adopted the stellar- or brown dwarf status of the objects as given in the literature. The broad range of age estimates for the nearby brown dwarfs (a few $100 \mathrm{Myr}$ to about $10 \mathrm{Gyr}$ ) and the substellar mass-age-luminosity degeneracy imply that masses cannot be obtained accurately, except for short period binaries, for which dynamical masses can be measured. To simplify, we considered i) a solar metallicity, stellar-brown dwarf boundary mass (0.075 $M_{\odot}$, Chabrier \& Baraffe 2000); (ii) all substellar objects of Table 1 as part of the brown dwarf group, even if some might reach planetary masses, below the theoretical minimum mass for deuterium burning ( $\sim 0.013 M_{\odot}$, Saumon et al. 1996); and (iii) stars and brown dwarfs as two groups, without studying their mass overlap due to age, metallicity, or dynamical mass uncertainties further. Nevertheless, we recall that low-mass stars are predicted to reach effective temperatures $\gtrsim 2000 \mathrm{~K}$ (as estimated using the 10 Gyr Dusty model from Chabrier et al. 2000, and corresponding to optical spectral types $\lesssim \mathrm{L} 3$, using the scale from Kirkpatrick et al. 2008) and that the cooler, late-type L, T, and $\mathrm{Y}$ dwarfs of the sample are therefore expected to be substellar objects, independent of age (see also Kirkpatrick 2013). The L7.5 and T0.5 components of WISE J1049-5319 in the $6.5 \mathrm{pc}$ sample, for instance, present Li I absorption in their optical spectra and are brown dwarfs (Faherty et al. 2014; Lodieu et al. 2015) based on the lithium test (Rebolo et al. 1992). Besides, LP 94420 (M9) is the unique M-type object in the 6.5 pc sample identified as a brown dwarf, also through the lithium test. Accounting for the most massive star, Sirius A (A1.0 V; $2.03 M_{\odot}$, Holberg et al. 2013), the stellar mass range is $2-0.075 M_{\odot}$; we note that the stellar dwarf with the latest spectral type, WISE J07200846 A (M9.5 V), has a dynamical mass estimate of $0.08 M_{\odot}$ (Burgasser et al. 2015b). Accounting for the least massive substellar object, WISE 0855-0714 (>Y2, Luhman 2014b), the substellar mass range is $0.075-0.007 M_{\odot}$.

We represent the 136 stars (including the Sun) and 26 brown dwarfs on the Galactic plane, in the left- and right-hand panels of Fig. 1, respectively. These stars distribute uniformly with about $50 \%$ ahead $(l=0-180 \mathrm{deg})$ and $50 \%$ behind $(l=180-360 \mathrm{deg})$ the Sun relative to the direction of Galaxy rotation $(l=90 \mathrm{deg})$. On the other hand, brown dwarfs do not distribute uniformly: there are $21(81 \%)$ behind and only five $(19 \%)$ ahead of the Sun (including the $\mathrm{Y}$ dwarf at $l=178 \mathrm{deg}$ ). Both the T- and Y-type brown dwarfs present a deficit at $l=0-180 \mathrm{deg}$ : four of the five $\mathrm{Y}$ dwarfs are at 180-360 deg, while there are $14 \mathrm{~T}$ dwarfs at 180-360 deg compared to only four T dwarfs at $l=0-180 \mathrm{deg}$. Even the two late-L- and the one late-M-type brown dwarfs are at $l=180-360 \mathrm{deg}$ (near $l \sim 270 \mathrm{deg}$ ). Besides this, the six (T-type) brown dwarfs that are companions to five stars (symbols with blue surroundings in Fig. 1) are also at $l=180-360 \mathrm{deg}$. 


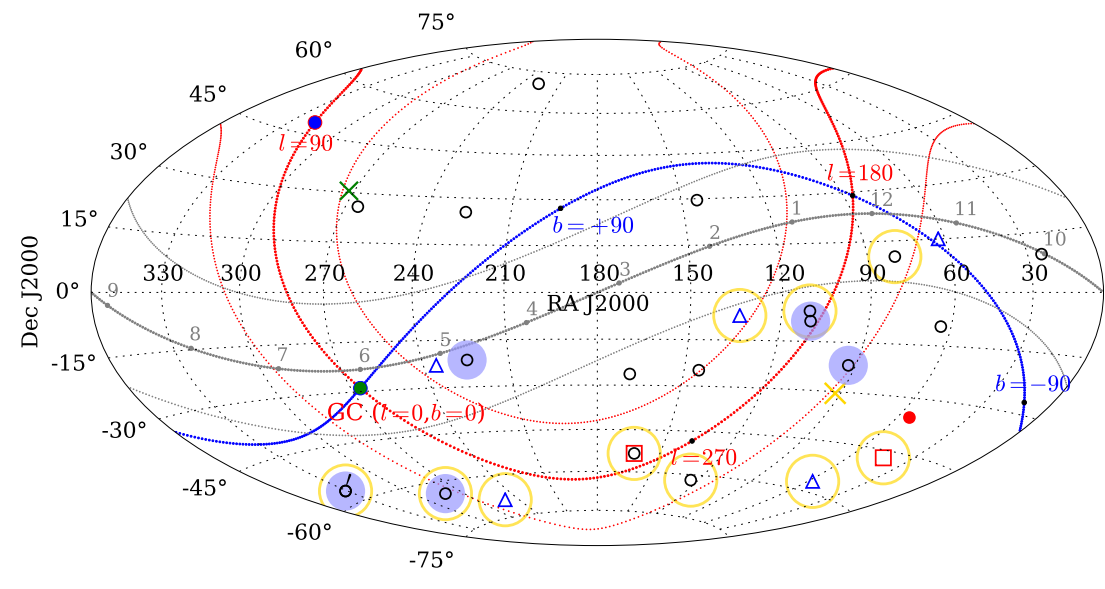

Fig. 2. Equatorial coordinate sky map in the Hammer equal-area projection of brown dwarfs at $<6.5 \mathrm{pc}$. Same symbols as in Fig. 1. The Galactic plane (with $b= \pm 20$ deg parallels) and its orthogonal plane along $l=0 \mathrm{deg}$ are represented by the red and blue lines, while the Galactic centre and the direction of Galactic rotation are indicated by the green and blue filled circles, respectively. The region that is particularly void of brown dwarfs is above the blue line ( $l \sim 0-180 \mathrm{deg})$. The solar apex and antapex are indicated by green and yellow crosses. The path of the Sun (ecliptic) with $\beta= \pm 20$ deg parallels are represented by the grey lines. The month (approximate mid-month date) of optimal night visibility from observatories such as at Mauna Kea or Cerro Paranal for each emphasized sky location on the ecliptic are indicated.
The same stars and brown dwarfs projected on the orthogonal plane to the Galactic plane and along $l=0$ deg (see Fig. A.1) distribute relatively uniformly. As in the previous projection, because the initial volume is a sphere, the space sampled transversally is smaller at larger radii, in contrast to a cylindrical volume, which explains the fewer objects. Among the nearest objects, $48.5 \%(66 / 136)$ of the stars belong to stellar multiple systems, while $15.4 \%(4 / 26)$ of the brown dwarfs belong to brown dwarf multiple systems (see Figs. 1 and A.1). At least five stars have brown dwarf companions and 10 stars other than the Sun have confirmed planets (based on the Open Exoplanet Catalogue $^{2}$ ), implying percentages of $>3.7$ and $>8.1 \%$, respectively. The number of substellar companions found to the stars and brown dwarfs will probably increase with the sensitivity of the low-mass companion searches.

Assuming a uniform distribution of brown dwarfs on the Galactic plane, as observed for stars and as also observed on the perpendicular plane for both brown dwarfs and stars, we would expect a symmetry between the two halves of the projected sphere, split by the galactocentric line crossing the Sun. The probability of the observed configuration can then be estimated using the binomial distribution $P(k ; n, p)=n ! /(k !(n-k) !)$ $* p^{k} *(1-p)^{n-k}=n ! /(k !(n-k) !) * 0.5^{n}$, where $k$ is the number of brown dwarfs ahead of the Sun, $n$ the total number of brown dwarfs, and $p=0.5$ the probability of an object being ahead of the Sun for the assumed symmetric distribution. Thus $P(5 ; 26)=0.09802 \%$. Excluding the six brown dwarf companions to stars, the configuration probability for the substellar population unbound to stars would be $P(5 ; 20)=1.47858 \%$.

Subtracting or adding the uncertainties to the parallaxes implies larger or smaller distances and thus may decrease or increase the number of objects within $6.5 \mathrm{pc}$, respectively. Because some parallaxes may present larger errors than those quoted (e.g. for preliminary trigonometric parallaxes based on few epochs or objects that are multiple and thus farther than the assumed single-object spectrophotometric distance), we considered subtracting or adding twice the quoted uncertainty. In the first case, the space ahead of the Sun is left void $(k=0)$, the front Y0-type WISE J0410+1502, and the four T-type WISE J1506+ 7027, WISE J1741+2553, 2MASS J1503+2525, and SIMP J0136+ 0933 having shifted out of the $<6.5 \mathrm{pc}$ volume, while $n=18$ brown dwarfs remain behind the Sun (two T- and the one M-type dwarf shifting out, too). In the second case, 12 objects shift in $(n=38)$, nine ahead (a), and three behind (b) the Sun $(k=14)$, namely WISE J0313+7807, WISE J2056+1459, and

\footnotetext{
www. openexoplanetcatalogue.com, as on 2016 February 9.
}

WISE J2209+2711 (T8.5, Y0, and Y1, -a-; trigonometric parallaxes from Beichman et al. 2014), WISE J1405+5534 and WISE J0254+0223 (Y0 and T8, -a-; trigonometric parallaxes from Dupuy \& Kraus 2013), WISE J1928+2356, WISE J0005+3737, WISE J0049+2151, WISE J0607+2429, and 2MASS J03486022 (T6, T9, T8.5, L9, and T7, -aaabb-; spectro-photometric distances from Kirkpatrick et al. 2012, where we assume $1 \sigma$ errors of $1 \mathrm{pc}$ ), and WISE J2030+0749 and WISE J0457-0207 (T1.5 and T2, -ab-; spectro-photometric distances from Bihain et al. 2013). Assuming that the distance estimates of the objects are independent, we can compare the minimum number of objects ahead $(k=0)$ to the maximum number of objects behind (24) to obtain the lower limit probability, $P(0 ; 24)=$ $0.000006 \%$. Conversely, we can compare the maximum number of objects ahead $(k=14)$ to the minimum number of objects behind $(18)$ to obtain the higher limit probability, $P(14 ; 32)=$ $10.97647 \%$.

Therefore, the observed brown dwarf configuration would have a probability with $2 \sigma$ error bars of $0.098_{-0.098}^{+10.878} \%$.

Finally, the five brown dwarfs at $l=0-180 \mathrm{deg}$ are farther at 5.2-6.5 pc, implying the brown dwarf distribution at $\lesssim 5 \mathrm{pc}$ is even less uniform (as highligted in Figs. 1 and A.1). Indeed, at less than the half-volume distance of $5.16 \mathrm{pc}$, the brown dwarf numbers reduce to zero and 12 ahead and behind the Sun, respectively, compared to five and nine at 5.16-6.5 pc in the outer shell volume, while the stellar distribution remains relatively uniform. Furthermore, at $<5.16 \mathrm{pc}$, the brown dwarfs are close to or below the Galactic height of the Sun. Extending the $6.5 \mathrm{pc}$ sample to a less complete and less accurate 8 pc sample by including farther brown dwarfs (as above), we find eight and three additions ahead and behind the Sun, and thus in total 13 and 24 brown dwarfs, respectively, indicating about half less substellar objects ahead of the Sun $(P(13 ; 37)=2.592 \%)$. Using the initial $8 \mathrm{pc}$ data of Kirkpatrick et al. (2012), we similarly find 12 and 21 brown dwarfs.

\section{Discussion}

To discuss the brown dwarf distribution further, we represent these objects in equatorial coordinates on a Hammer equal-area projection, as shown in Fig. 2. Here also we see a clear void that is well defined above the blue line (orthogonal plane to the Galactic plane, along $l=0 \mathrm{deg}$ ). It coincides in part with the northern hemisphere. Furthermore, there are no brown dwarfs found yet within $-20<b<20$ deg of the Galactic plane (between the two outer red lines) and at $l=0-180 \mathrm{deg}$.

We wonder whether the non-uniform distribution of the brown dwarfs at $<6.5 \mathrm{pc}$ could be related to (i) a small number 


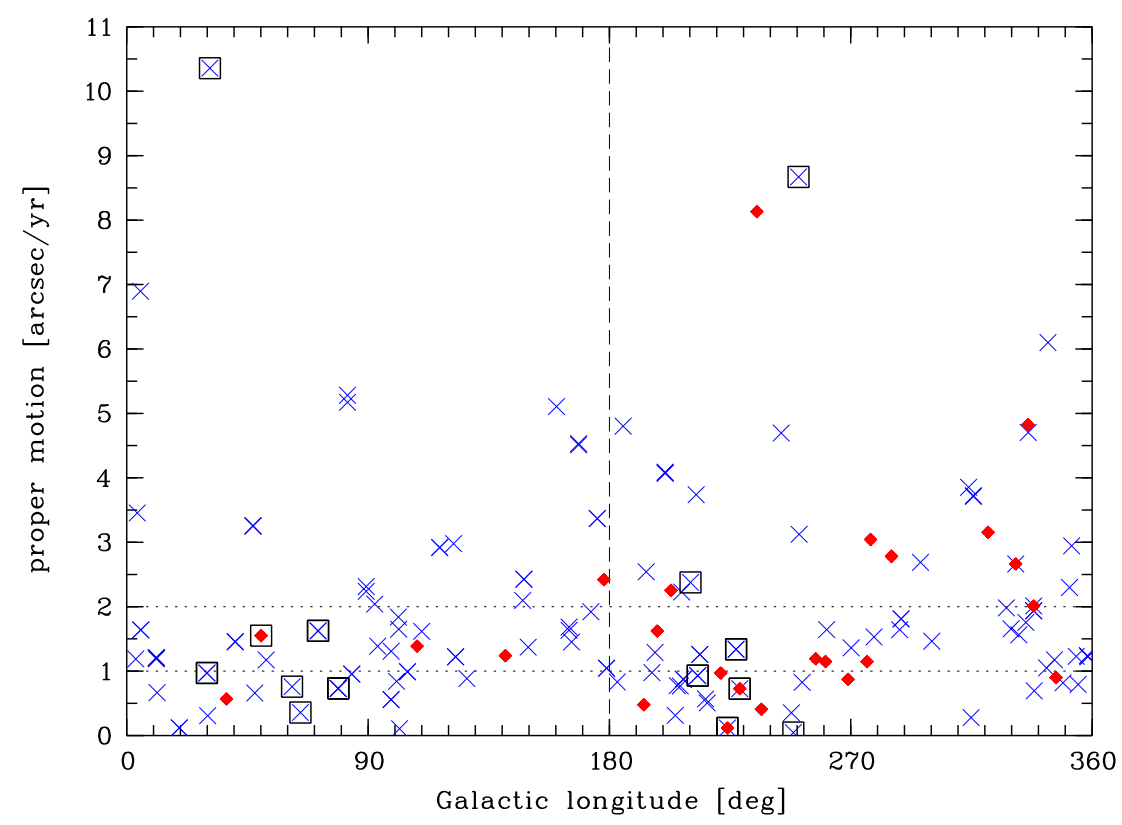

Fig. 3. Proper motions of 135 stars (crosses) and 26 brown dwarfs (filled lozenges) in the $6.5 \mathrm{pc}$ sample as a function of Galactic longitude. The dashed line marks the dividing line between the two hemispheres front and back of the Sun relative to the direction of Galactic rotation. The brown dwarf distribution in the left part differs from that in the right part, with a deficit at low ( $\$ 1 \mathrm{arcsec} / \mathrm{yr})$ and high $(\gtrsim 2 \operatorname{arcsec} / \mathrm{yr})$ proper motions indicated by dotted lines. Open squares indicate objects within $30 \mathrm{deg}$ of the solar apex and antapex, respectively. bias; (ii) incompleteness of all-sky surveys; (iii) incompleteness of the searches; and (iv) "brown dwarf" statistics or Galactic dynamics:

(i) While the number of brown dwarfs is small when divided into spectral classes (1M, 2L, 18T, and 5Y), the total number (26) is large and representative enough for statistical estimations, and it indicates that the distribution is remarkably non-uniform. We note that the $17 \mathrm{~K}$-type dwarfs, almost equal in number to the $18 \mathrm{~T}$ dwarfs, do not distribute uniformly over the whole projected sphere either, with no object farther than $2 \mathrm{pc}$ behind the $l=0$ deg line; however, these stars distribute almost equally with nine ahead and eight behind the Sun. Nevertheless, and until the deepest and most accurate searches have fully scrutinized the 6.5 pc-radius sphere (or larger spheres), we suggest that the number of brown dwarfs may be incomplete.

(ii) All-sky surveys such as 2MASS and WISE are complete over most of the sky. However, in the densest regions towards the Galactic centre and plane and in globular clusters, there is confusion noise from too many sources, which reduce the survey depth limit, for example by about 1.5-2 mag for $2 \mathrm{MASS}^{3}$; besides this, bright stars with saturated counts, diffraction spikes, and halos complicate or impede the automatic selection and visual verification of candidates. However, an asymmetry with respect to the distribution of the problematic crowded regions in the two hemispheres separated by the blue dividing line in Fig. 2 is not expected.

(iii) While searching for nearby late-type dwarfs by photometry and sky motions using WISE and 2MASS, Kirkpatrick et al. (2014) found objects missed by Luhman (2014a), and conversely. The most notable example is their second highest motion object, the $J=9.0 \mathrm{mag}$ M7 dwarf WISE J1540-5101 at 4.4 pc (Kirkpatrick et al. 2014; Pérez Garrido et al. 2014). Therefore, even while recovering all previously known nearby dwarfs, independent searches find different new candidates. Kirkpatrick et al. (2014) point out that they were able to measure motions with enough accuracy only for the fastest among the nearest TY-type dwarfs,

3 www.astro.caltech.edu/ jmc/2mass/v3/gp/analysis.html because the apparent magnitudes of the dwarfs are so faint that their astrometric position errors at different epochs are very large. The more recent search by Schneider et al. (2016) gather a sample of new objects of not-so-small motions and about two magnitudes fainter (W2 $14 \mathrm{mag})$ than their previous sample (Kirkpatrick et al. 2014), however the late-type candidates are farther than $8 \mathrm{pc}$ from the Sun. Using the $W 2$-band survey limit of $14.5 \mathrm{mag}$, they rule out other objects as faint as WISE J0855-0714 at $\$ 2.9$ pc or new earlier Y-type dwarfs at $\$ 9.5 \mathrm{pc}$, and with proper motions of $0.25-15 \operatorname{arcsec} \mathrm{yr}^{-1}$. Brighter, new T-type dwarfs at short distances would then be ruled out even more.

Considering the proper motions of the stars and brown dwarfs in the 6.5 pc sample as a function of Galactic longitude, we find a deficit of both small and large proper motions for the brown dwarfs with $l \sim 0-180 \mathrm{deg}$ (see Fig. 3). The median proper motion is always (for stars and brown dwarfs in both hemispheres) at about $1.5 \mathrm{arcsec} / \mathrm{yr}$. However, the mean and the standard deviation are about 2.0 and $1.8 \mathrm{arcsec} / \mathrm{yr}$, respectively, except for the only five brown dwarfs at $l \sim 0-180 \mathrm{deg}$, for which these values are reduced to 1.4 and 0.7 arcsec/yr, respectively. In addition to the deficit of low and very high proper motion brown dwarfs at $l \sim 0-180 \mathrm{deg}$, some brown dwarfs (and stars) with relatively small proper motions near the solar apex- $(l=57, b=+22 \mathrm{deg})$ and antapex directions $(l=237, b=-22 \mathrm{deg})$ could still be missing 4 .

Thus, new brown dwarfs at $<6.5 \mathrm{pc}$ (especially at $l=0-180 \mathrm{deg}$ and $\lesssim 5 \mathrm{pc}$ ) may still be discovered eventually (a) at both small ( $\$ 1$ arcsec/yr) and large ( $~ 2 \operatorname{arcsec} / y r)$ proper motions or (b) in the extremely crowded regions of the Galactic plane, or any sky location where blends or mismatches with unrelated sources or discarded matches with related sources can occur. A near-infrared survey 3 to 4 mag deeper than 2MASS such as the Visible and Infrared Survey Telescope for Astronomy (VISTA) south - Hemisphere Survey and the UKIRT Infrared Deep

4 This does not impede the two highest proper motion stars in the sky, Barnard's and Kapteyn's stars, to be near the apex and antapex, respectively. 
Sky Survey (UKIDSS; see e.g. discoveries by Warren et al. 2007; Burningham et al. 2009, 2011; Scholz 2010a,b; Goldman et al. 2010; Lucas et al. 2010; Pinfield et al. 2012; Lodieu et al. 2012; Gauza et al. 2015), extended to the complete northern hemisphere, could be propitious. Ultimately, some bright candidates might not be followed up spectroscopically yet, because of a lack of timely observability. Figure 2 shows that the $l=0-90$ deg Galactic quadrant is accessible to night observation during spring and summer, whereas the $l=90-180$ deg Galactic quadrant is accessible during autumn and winter, from a northern observatory, such as at Mauna Kea.

(iv) If this observed non-uniform substellar distribution remains unchanged after future searches, then either some fortuitous random aggregation or some dynamics affecting primarily the lower mass objects, could be invoked. We note that pencil beam deep surveys of the Milky Way suggests there might be slightly more M-type dwarfs in the Galactic northern hemisphere $(b>0 \mathrm{deg})$ than in the southern hemisphere $(b<0$ deg; see Holwerda et al. 2014 and references therein) and hints at a reversal occuring for midto late M-type dwarfs, which would be more numerous in the southern hemisphere. However, as already mentioned in Sect. 2 and shown in Fig. A.1, the objects in our sample distribute uniformly perpendicular to the Galactic plane; indeed, we count 50 and $51 \mathrm{M}$ dwarfs at $b \geq 0$ and $<0$ deg, respectively, and equal numbers of brown dwarfs. This is not surprising since the size of our sample (13 pc diameter) is at least a few tens times smaller than typical disk scale heights. The cause of the planar front/back asymmetry observed for the brown dwarfs is thus likely unrelated to that of the large scale perpendicular asymmetry for stars, the latter asymmetry also derived from the Sloan Digital Sky Survey (SDSS) or RAdial Velocity Experiment survey (RAVE) catalogues and attributed to wavelike gravitational perturbations (Widrow et al. 2012; Yanny \& Gardner 2013; Williams et al. 2013).

\section{Conclusions}

For the updated $<6.5 \mathrm{pc}$ sample, we find a star-to-brown-dwarf ratio of $136 / 26=5.2$, while Kirkpatrick et al. (2012) find a ratio of 6 (8 pc sample), and Henry et al. (2016) count 10 times more stars than brown dwarfs ${ }^{5}$ (in their RECONS 10 pc sample). Because of the heliocentric and geocentric nature of the distribution at $<6.5 \mathrm{pc}$, the non-uniformity of the substellar distribution on the Galactic plane is likely due to an observational bias (since we expect brown dwarfs to distribute uniformly as stars do). The brown dwarf census would thus be incomplete. Assuming five more brown dwarfs ahead of the Sun, i.e. the addition of twothirds of the observed excess relative to uniformity, the star-tobrown-dwarf ratio would decrease to $136 / 31=4.4_{-0.7}^{+1.0}$, considering error bars corresponding to Poissonian uncertainties of the number of brown dwarfs. It is slightly higher but agrees with the ratio $N\left(0.08-1.0 M_{\odot}\right) / N\left(0.03-0.08 M_{\odot}\right)=3.3_{-0.7}^{+0.8}$ estimated in the Orion Nebula cluster (Slesnick et al. 2004; Andersen et al. 2008), noting also that the multiplicity of the nearby stars may be better accounted for than in Orion (higher number of stars). As already pointed out by Kirkpatrick et al. (2012), the starto-brown-dwarf ratii of the solar neighbourhood and star forming regions may also differ because they consist of different

\footnotetext{
5 Abstract for AAS Meeting in Kissimmee, FL in January 2016, available at RECONS web page.
}

dynamical environments with different star formation histories and mass segregations. In the much less probable case the relative void of substellar objects at $l \sim 0-180 \mathrm{deg}$ (or excess at $l \sim 180-360 \mathrm{deg}$ ) is real, this could be related to some random inhomogeneity or dynamical effect of trapping or deflection taking place for small objects, so that they would be located more behind than ahead of the Sun relative to the direction of the rotation of the Galaxy.

Acknowledgements. We thank the anonymous referee for a constructive review that helped us to improve the paper. This research made use of the VizieR catalogue access tool and the SIMBAD database provided by the CDS Strasbourg, France, the M, L, T, and Y dwarf compendium housed at www . DwarfArchives. org, and the solar neighbourhood census provided by RECONS (www . recons. org).

\section{References}

Andersen, M., Meyer, M. R., Greissl, J., \& Aversa, A. 2008, ApJ, 683, L183 Artigau, É., Doyon, R., Lafrenière, D., et al. 2006, ApJ, 651, L57

Artigau, É., Radigan, J., Folkes, S., et al. 2010, ApJ, 718, L38

Beichman, C., Gelino, C. R., Kirkpatrick, J. D., et al. 2014, ApJ, 783, 68

Béjar, V. J. S., Martín, E. L., Zapatero Osorio, M. R., et al. 2001, ApJ, 556, 830 Bihain, G., Scholz, R.-D., Storm, J., \& Schnurr, O. 2013, A\&A, 557, A43

Burgasser, A. J., Geballe, T. R., Leggett, S. K., Kirkpatrick, J. D., \& Golimowski, D. A. 2006, ApJ, 637, 1067

Burgasser, A. J., Tinney, C. G., Cushing, M. C., et al. 2008, ApJ, 689, L53 Burgasser, A. J., Sheppard, S. S., \& Luhman, K. L. 2013, ApJ, 772, 129 Burgasser, A. J., Gillon, M., Melis, C., et al. 2015a, AJ, 149, 104 Burgasser, A. J., Melis, C., Todd, J., et al. 2015b, AJ, 150, 180 Burningham, B., Pinfield, D. J., Leggett, S. K., et al. 2009, MNRAS, 395, 1237 Burningham, B., Lucas, P. W., Leggett, S. K., et al. 2011, MNRAS, 414, L90 Chabrier, G., \& Baraffe, I. 2000, ARA\&A, 38, 337

Chabrier, G., Baraffe, I., Allard, F., \& Hauschildt, P. 2000, ApJ, 542, 464 Costa, E., Méndez, R. A., Jao, W.-C., et al. 2006, AJ, 132, 1234 Cushing, M. C., Kirkpatrick, J. D., Gelino, C. R., et al. 2011, ApJ, 743, 50 Dieterich, S. B., Henry, T. J., Jao, W.-C., et al. 2014, AJ, 147, 94 Dupuy, T. J., \& Kraus, A. L. 2013, Science, 341, 1492

Dupuy, T. J., \& Liu, M. C. 2012, ApJS, 201, 19

Faherty, J. K., Beletsky, Y., Burgasser, A. J., et al. 2014, ApJ, 790, 90

Finch, C. T., Zacharias, N., Subasavage, J. P., Henry, T. J., \& Riedel, A. R. 2014, AJ, 148, 119

Gaidos, E., Mann, A. W., Lépine, S., et al. 2014, MNRAS, 443, 2561

Gauza, B., Béjar, V. J. S., Pérez-Garrido, A., et al. 2015, ApJ, 804, 96 Giammichele, N., Bergeron, P., \& Dufour, P. 2012, ApJS, 199, 29

Goldman, B., Marsat, S., Henning, T., Clemens, C., \& Greiner, J. 2010, MNRAS, 405, 1140

Henry, T. J., Subasavage, J. P., Brown, M. A., et al. 2004, AJ, 128, 2460

Henry, T. J., Jao, W.-C., Subasavage, J. P., et al. 2006, AJ, 132, 2360

Holberg, J. B., Oswalt, T. D., Sion, E. M., Barstow, M. A., \& Burleigh, M. R. 2013, MNRAS, 435, 2077

Holwerda, B. W., Trenti, M., Clarkson, W., et al. 2014, ApJ, 788, 77

Ivanov, V. D., Vaisanen, P., Kniazev, A. Y., et al. 2015, A\&A, 574, A64

Kasper, M., Biller, B. A., Burrows, A., et al. 2007, A\&A, 471, 655

Kirkpatrick, J. D. 2013, Astron. Nachr., 334, 26

Kirkpatrick, J. D., Cruz, K. L., Barman, T. S., et al. 2008, ApJ, 689, 1295

Kirkpatrick, J. D., Cushing, M. C., Gelino, C. R., et al. 2011, ApJS, 197, 19

Kirkpatrick, J. D., Gelino, C. R., Cushing, M. C., et al. 2012, ApJ, 753, 156

Kirkpatrick, J. D., Schneider, A., Fajardo-Acosta, S., et al. 2014, ApJ, 783, 122 Leggett, S. K., Saumon, D., Marley, M. S., et al. 2012, ApJ, 748, 74

Leggett, S. K., Morley, C. V., Marley, M. S., \& Saumon, D. 2015, ApJ, 799, 37 Lodieu, N., Burningham, B., Day-Jones, A., et al. 2012, A\&A, 548, A53

Lodieu, N., Zapatero Osorio, M. R., Rebolo, R., et al. 2015, A\&A, 581, A73 Lucas, P. W., Tinney, C. G., Burningham, B., et al. 2010, MNRAS, 408, L56 Luhman, K. L. 2013, ApJ, 767, L1

Luhman, K. L. 2014a, ApJ, 781, 4

Luhman, K. L. 2014b, ApJ, 786, L18

Luhman, K. L., \& Esplin, T. L. 2014, ApJ, 796, 6

McCaughrean, M. J., Scholz, R.-D., \& Lodieu, N. 2002, A\&A, 390, L27

McCaughrean, M. J., Close, L. M., Scholz, R.-D., et al. 2004, A\&A, 413, 1029

Mužić, K., Scholz, A., Geers, V. C., \& Jayawardhana, R. 2015, ApJ, 810, 159

Nakajima, T., Oppenheimer, B. R., Kulkarni, S. R., et al. 1995, Nature, 378, 463

Oppenheimer, B. R., Kulkarni, S. R., Matthews, K., \& Nakajima, T. 1995, Science, 270, 1478 
Peña Ramírez, K., Béjar, V. J. S., Zapatero Osorio, M. R., Petr-Gotzens, M. G. \& Martín, E. L. 2012, ApJ, 754, 30

Peña Ramírez, K., Béjar, V. J. S., \& Zapatero Osorio, M. R. 2016, A\&A, 586, A157

Pérez Garrido, A., Lodieu, N., Béjar, V. J. S., et al. 2014, A\&A, 567, A6

Pinfield, D. J., Burningham, B., Lodieu, N., et al. 2012, MNRAS, 422, 1922

Rebolo, R., Martín, E. L., \& Magazzù, A. 1992, ApJ, 389, L83

Saumon, D., Hubbard, W. B., Burrows, A., et al. 1996, ApJ, 460, 993

Schilbach, E., Röser, S., \& Scholz, R.-D. 2009, A\&A, 493, L27

Schneider, A. C., Cushing, M. C., Kirkpatrick, J. D., et al. 2015, ApJ, 804, 92

Schneider, A. C., Greco, J., Cushing, M. C., et al. 2016, ApJ, 817, 112

Scholz, R.-D. 2010a, A\&A, 515, A92

Scholz, R.-D. 2010b, A\&A, 510, L8

Scholz, R.-D. 2014, A\&A, 561, A113

Scholz, R.-D., Lo Curto, G., Méndez, R. A., et al. 2005, A\&A, 439, 1127
Scholz, R.-D., Bihain, G., Schnurr, O., \& Storm, J. 2011, A\&A, 532, L5

Skrutskie, M. F., Cutri, R. M., Stiening, R., et al. 2006, AJ, 131, 1163

Slesnick, C. L., Hillenbrand, L. A., \& Carpenter, J. M. 2004, ApJ, 610, 1045

Tinney, C. G. 1998, MNRAS, 296, L42

Tinney, C. G., Faherty, J. K., Kirkpatrick, J. D., et al. 2014, ApJ, 796, 39

van Leeuwen, F. 2007, A\&A, 474, 653

Warren, S. J., Mortlock, D. J., Leggett, S. K., et al. 2007, MNRAS, 381, 1400

Widrow, L. M., Gardner, S., Yanny, B., Dodelson, S., \& Chen, H.-Y. 2012, ApJ, 750, L41

Williams, M. E. K., Steinmetz, M., Binney, J., et al. 2013, MNRAS, 436, 101

Winters, J. G., Henry, T. J., Lurie, J. C., et al. 2015, AJ, 149, 5

Wright, E. L., Eisenhardt, P. R. M., Mainzer, A. K., et al. 2010, AJ, 140, 1868

Yanny, B., \& Gardner, S. 2013, ApJ, 777, 91

Zapatero Osorio, M. R., Gálvez Ortiz, M. C., Bihain, G., et al. 2014, A\&A, 568, A77

\section{Appendix A: Distribution of the nearest stars and brown dwarfs on the Galactic orthogonal plane}
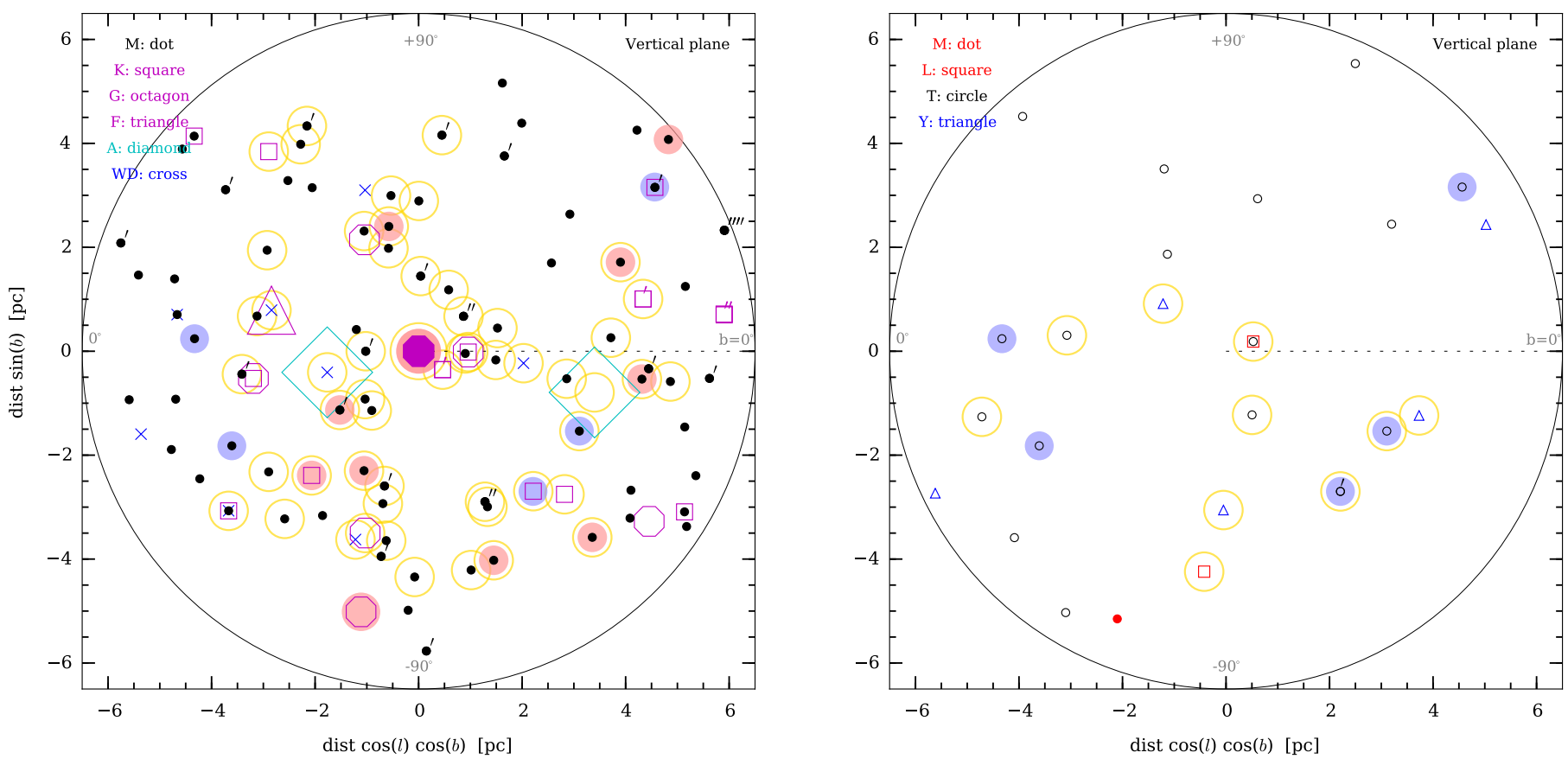

Fig. A.1. Same as Fig. 1, but on the Galactic orthogonal plane along $l=0$ deg. 